\title{
NEW STABLE, EXPLICIT, FIRST ORDER METHOD TO SOLVE THE HEAT CONDUCTION EQUATION
}

\author{
ENDRE KOVÁCS \\ Department of Physics and Electrical Engineering, University of Miskolc \\ H-3515 Miskolc-Egyetemváros, Hungary \\ kendre01@gmail.com
}

[Received: May 29, 2020, Accepted: June 12, 2020]

\begin{abstract}
In this paper a novel explicit and unconditionally stable numerical algorithm is introduced to solve the inhomogeneous non-stationary heat or diffusion equation. Spatial discretization of these problems usually yields huge and stiff ordinary differential equation systems, the solution of which is still time-consuming. The performance of the new method is compared with analytical and numerical solutions. It is proven exactly as well as demonstrated numerically that the new method is first order in time and can give approximate results for extremely large systems faster than the commonly used explicit or implicit methods. The new method can be easily parallelized and it is handy to apply regardless of space dimensions and grid irregularity
\end{abstract}

Mathematical Subject Classification: 65L04, 65N40, 80M25

Keywords: Heat equation, explicit methods, stable schemes, stiff equations

\section{INTRODUCTION}

It is well known that the simplest Fourier-type heat conduction phenomena are described by the heat equation, which is a second-order parabolic partial differential equation (PDE), with the following form.

$$
\frac{\partial T}{\partial t}=\alpha \nabla^{2} T+q
$$

where $\alpha=k /(c \rho)>0$ is the thermal diffusivity, $q, k, c$ and $\rho$ ares the volumetric intensity of heat sources (radiation, chemical reactions radioactive decay, etc.), heat conductivity, specific heat and (mass) density, respectively. This equation describes heat conduction in solid materials, not only in the walls of residential buildings but in different kinds of technical equipment like chimneys, heat exchangers and electronic circuits as well.

The PDEs for real-life problems can rarely be solved analytically. The new numerical procedure starts like the standard method of lines: the whole spatial domain is divided into smaller cells. However, this discretization must reflect the material properties of the system, thus we perform it more generally than numerical analysis textbooks usually do. We calculate three quantities for each cell. The first one is the heat capacity of the cell: $C=c m=c \rho V$, where $m$ is the mass, $V$ is the volume 
of the cell. Now the (thermal) energy of a cell can be expressed as $C_{i} T_{i}$, where $T_{i}$ is the average temperature of the cell. The second quantity is the heat or thermal conductance $U$, which can be approximated as

$$
U_{i j} \approx k \frac{A_{i j}}{d_{i j}},
$$

where $A_{i j}$ is the surface area between the two cells $i$ and $j$, while $d_{i j}$ is the distance between the centres of the cells. In a one dimensional non-uniform grid, $d_{i j}=\left(\Delta x_{i}+\Delta x_{j}\right) / 2$. The third quantity is $Q_{i}$, the heat source term:

$$
Q_{i}=\frac{1}{V_{i}} \int_{V_{i}} q \mathrm{dV}, \quad \text { in }\left[\frac{\mathrm{K}}{\mathrm{s}}\right] \text { units. }
$$

After the discretization of the second spatial derivatives according to the most common second order central difference formula, we obtain an ordinary differential equation (ODE) system which gives the time derivative of each cell's temperature

$$
\frac{\mathrm{d} T_{i}}{\mathrm{dt}}=\sum_{j=n e i g h} \frac{U_{i j}}{C_{i}}\left(T_{j}-T_{i}\right)+Q_{i},
$$

where the summation is carried out for the neighboring the cells. We can write it in a matrix form:

$$
\frac{\mathrm{d} \vec{T}}{\mathrm{dt}}=M \vec{T}+\vec{Q}
$$

To help the reader visualize, we present the arrangement of the variables in Figure 1 for a $2 D$ system of 3 cells.

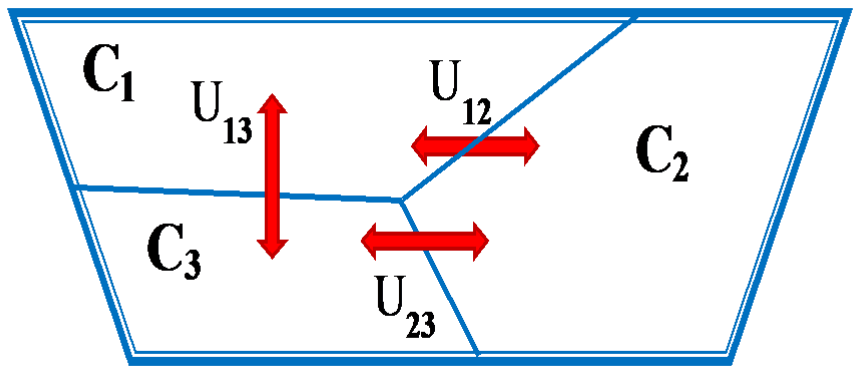

Figure 1. Notations in the case of three cells. The outer double line represents thermal isolation. Note that the shape and arrangement of the cells are not necessarily regular. 
The ODE system in a matrix form for this small system:

$$
\frac{\mathrm{d} \vec{T}}{\mathrm{dt}}=\left[\begin{array}{ccc}
-\frac{U_{12}}{C_{1}}-\frac{U_{13}}{C_{1}} & \frac{U_{12}}{C_{1}} & \frac{U_{13}}{C_{1}} \\
\frac{U_{12}}{C_{2}} & -\frac{U_{12}}{C_{2}}-\frac{U_{23}}{C_{2}} & \frac{U_{23}}{C_{2}} \\
\frac{U_{13}}{C_{3}} & \frac{U_{23}}{C_{3}} & -\frac{U_{13}}{C_{3}}-\frac{U_{23}}{C_{3}}
\end{array}\right] \vec{T}+\left[\begin{array}{c}
Q_{1} \\
Q_{2} \\
Q_{3}
\end{array}\right]
$$

Suppose now that one quickly needs approximate results about the temperature distribution as a function of time in an extremely large and complicated system, where the physical properties of the material like the specific heat and the thermal diffusivity widely vary from point to point. In this case the size of the matrix is huge, while the magnitude of the matrix elements and that of the eigenvalues has a range of several orders of magnitude, which means it is an extremely stiff system. Which method can be recommended to solve this problem?

It is widely believed that conventional explicit methods are inappropriate because of unacceptably small timesteps due to stiffness $[1,2$. That is why implicit methods are almost exclusively used in the industry [3], as they have excellent stability properties. The famous book of Hairer and Wanner 4 even devotes a separate, second volume to stiff (and differential-algebraic) problems, where they almost exclusively deal with implicit methods. The disadvantage of the implicit methods is that they require the solution of an algebraic equation system at each time-step, predominantly by iterative methods, which is slow because of the necessity to store and handle huge matrices. Moreover, parallelization of implicit methods is not straightforward, although some moderate progress has been achieved [5 7]. However, as the clock frequency increase of CPUs has significantly slowed down in the last two decades, parallel programming has become the dominant paradigm in high performance computing $[8$. The easily parallelizable explicit methods with better stability properties like Runge-Kutta-Chebyshev, ADE (Alternating Direction Explicit), Hopscotch or Dufort-Frankel methods are very rarely used, as they usually have other disadvantages: they can hardly be applied for irregular grids, they can be rather inaccurate, complicated to implement, or they can only be conditionally consistent $[2,9,13]$.

We have to conclude that conventional methods provide no convenient solution. In order to fill this gap and solve these systems more effectively, we started to elaborate a family of fundamentally new explicit methods. The simplest version for transient problems (i.e. when $q \equiv 0$ ) has already been published [14]. The version of the algorithm and the logic by which we obtained that algorithm was not suitable for further work, i.e. for the generalization of the method. Moreover, 14 contains no verification by comparing the results to analytical solutions, and the proof for the rate of the convergence is presented only in the current paper. The numerical results presented here are for a larger and stiffer system than those shown in [14]. 


\section{THE PROPOSED METHOD}

Now we introduce the core method to solve the ODE system (1) through the following two steps:

1. We make a simplification: when we calculate the new value of a variable $T_{i}$, we neglect the fact that other variables are also changing during the timestep. This means that we consider $T_{j}$ a constant if $j \neq i$, thus we can call it "constantneighbour method", abbreviated by CN. So we have to solve uncoupled, linear ODEs:

$$
T_{i}^{\prime}=a_{i}-T_{i} / \tau_{i}
$$

where

$$
a_{i}=\sum_{j \neq i} m_{i j} T_{0, j}+Q_{i}
$$

is considered as a constant. We also introduce

$$
\tau_{\mathrm{i}}=-\frac{1}{m_{i i}}=\frac{C_{i}}{\sum_{j=n e i g h} U_{i j}},
$$

which is a time-constant for the cells, while $T_{0, j}$ is the initial temperature.

2. The equations obtained are solved analytically. The analytical solution of equation (2) is given by

$$
T_{i}(t)=T_{0, i} e^{-\frac{t}{\tau_{i}}}+a_{i} \tau_{i}\left(1-e^{-\frac{t}{\tau_{i}}}\right) .
$$

Thus the following simple formula is proposed to obtain the values of $T$ at the end of the first timestep using the values of $T_{0, i}$ only at the beginning of the timestep:

$T_{i}(h)=\left\{T_{i b} e^{-\frac{h}{\tau_{i}}}+\frac{\sum_{j=n e i g h} U_{i j} T_{0, j}}{\sum_{j=n e i g h} U_{i j}}\left(1-e^{-\frac{h}{\tau_{i}}}\right)\right\}+Q_{i} \tau_{i}\left(1-e^{-\frac{h}{\tau_{i}}}\right)$.

Using the identity $e^{x}=1+\left(e^{x}-1\right)$ and the definition of $\tau$, we can write (3) into the following form

$$
\begin{aligned}
T_{i}(h)=T_{0, i}+\left\{\frac{m_{i i}}{T_{0, i}} m_{i i}+\frac{\sum_{j \neq i} m_{i j} T_{0, j}}{m_{i i}}+\frac{Q_{i}}{m_{i i}}\right\}\left(e^{m_{i i} h}-1\right)= \\
=T_{0, i}+\left(\sum_{j} m_{i j} T_{0, j}+Q_{i}\right) \frac{e^{m_{i i} h}-1}{m_{i i}} .
\end{aligned}
$$

Now one can see that this algorithm looks similar to the explicit Euler method, which would give:

$$
\vec{T}(h)=\vec{T}_{0}+h\left(M \vec{T}_{0}+\vec{Q}\right),
$$


which shows than in our case $\left(e^{m_{i i} h}-1\right) / m_{i i}$ stand for $h$. Using the power series of the exponential function, we can write

$$
\left(e^{m_{i i} h}-1\right) / m_{i i}=h+m_{i i} \frac{h^{2}}{2}+\ldots
$$

which means that, regarding one timestep, the new method is identical to the explicit Euler method up to first order. However, because of the exponential terms, formula (3) contains $h$ up to infinite order, which is crucial for the unconditional stability.

The first two terms on the right hand side of (3), in curly brackets describe the transient process. As physically justifiable, the temperature of each cell exponentially tends to the temperature of its neighbors: the new value of the variable $T_{i}$ is the weighted average of the old value of $T_{i}$ and its neighbors $T_{j}$. It is easy to see that the coefficients of the initial temperatures $T_{0, j}$ are non-negative and the sum of them is 1 . That is why the result is always bounded: the method cannot be unstable for the heat conduction equation. It is also obvious that the new method is explicit, one can calculate the new values without solving a system of equations or even without using matrices. It also implies that the process is obviously parallelizable and hopefully even vectorizable. Moreover, it can be easily applied regardless of the number of space dimensions, grid irregularity or inhomogeneity of the heat conduction medium. To examine the performance of the method, several numerical tests have been made, but here we present only two different examples.

\section{VERIFICATION: COMPARISON WITH AN EXACT RESULT}

If there are only two variables and one heat source, equation (1) has the following analytical solution

$$
\begin{gathered}
T_{1}(t)=T_{0,1} \exp (-t / \tau)+T_{\mathrm{a}}(1-\exp (-t / \tau))+S t+S \tau \frac{C_{2}}{C_{1}}(1-\exp (-t / \tau)), \\
T_{2}(t)=T_{0,2} \exp (-t / \tau)+T_{\mathrm{a}}(1-\exp (-t / \tau))+S t-S \tau(1-\exp (-t / \tau))
\end{gathered}
$$

where

$$
\tau=\frac{C_{1} C_{2}}{U\left(C_{1}+C_{2}\right)}, \quad T_{\mathrm{a}}=\frac{T_{0,1} C_{1}+T_{0,2} C_{2}}{C_{1}+C_{2}} \quad \text { and } \quad S=\frac{Q_{1}}{C_{1}+C_{2}}
$$

are the common time constant, the final temperature without external source (weighted average of the initial temperatures) and the common increment of the temperatures due to the $Q_{1}$ source in one time unit, respectively. The results obtained by the new method fit the exact values very well at the final time $t_{\text {fin }}$. An example for the errors as a function of the stepsize is presented in Figure 2. Error here means the absolute value of the difference between the exact temperature and the temperature obtained by the new method $\left|T^{\mathrm{ex}}\left(t_{\text {fin }}\right)-T^{\mathrm{CN}}\left(t_{\text {fin }}\right)\right|$. 


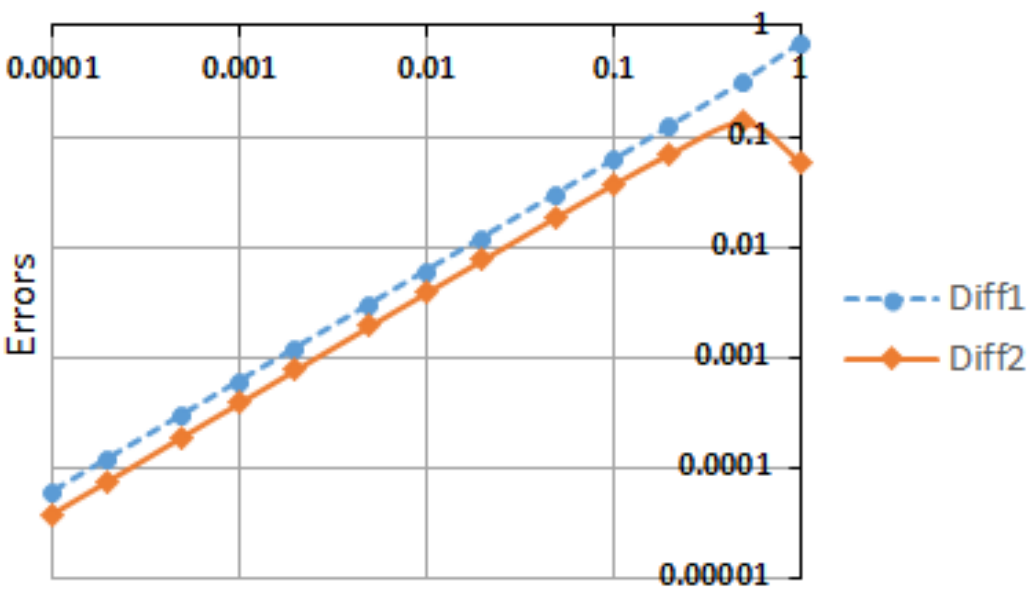

Time-step size $h$

Figure 2. Absolute value of errors as a function of the timestep size for $U=0, C_{1}=5 C_{2}=1, T_{01}=10, T_{02}=0, Q_{1}=8$ and $t_{\text {fin }}=1$. Diff1 is for the first, Diff2 is for the second cell.

It is well known that a method is called $p^{t h}$ order if the local error is $O\left(h^{p+1}\right)$ or (equivalently for normal systems) if the global error is $O\left(h^{p}\right)$. It can be seen from Figure 2 that the global error decreases with the first power of the stepsize, thus one can conclude that this method is first order. The analytical proof of this statement is presented in the Appendix.

\section{COMPARISON With NUMERICAL RESUlts FOR A STIFF SYSTEM}

The second system is a regular rectangle-shaped lattice, $N_{x}=100, N_{y}=50$. A value $10^{(3-7 \times \text { rand })}$ is given to the capacities $C_{i}$ and to the inverse conductances $1 / U_{x i}$, $1 / U_{y i}$ (the resistances), where rand is a random number generated by MATLAB uniformly in the $(0,1)$ interval for each quantity. This means that the capacities (the resistances) follow a log-uniform distribution between 0.0001 and 1000 . The initial temperatures are uniformly zero, the sources have a uniform distribution between 0 and $100, Q_{i}=100 \times(1-$ rand $)$. The task is to solve this system for the temperatures between $t_{0}=0 \mathrm{~s}$ and $t_{\mathrm{FIN}}=10 \mathrm{~s}$.

It is well known that the stiffness ratio is the ratio of the extreme eigenvalues $\lambda_{\min } / \lambda_{\max }$, where $\lambda_{\min }\left(\lambda_{\max }\right)$ is the (negative) eigenvalue of the matrix with the largest (smallest) absolute value. In this case the stiffness ratio is $7.6 \times 10^{12}$, which means that this is a seriously stiff problem. For the explicit Euler method (which is equivalent to the forward-time central-space FTCS scheme), the maximum possible timestep is

$$
h_{\mathrm{MAX}}^{\mathrm{E}}=\left|2 / \lambda_{\max }\right|=1.45 \times 10^{-8} s .
$$


Note that above this threshold instability necessarily occurs. For this system, the widely used (conditionally stable) explicit methods are inpractical, as they would require at least a day to reach any result. Therefore, to provide a reference solution, an implicit ode15s solver of MATLAB has been used, which is variable-step, variableorder, based on the numerical differentiation formulas (NDFs) of orders 1 to 5 , where the letter $s$ indicates that the codes were designed especially for stiff systems. With strict error tolerance ('RelTol' $=10^{-8}$, 'AbsTol' $=10^{-7}$ ), our computer needs $677 \mathrm{~s}$ to give a high precision solution using this routine.

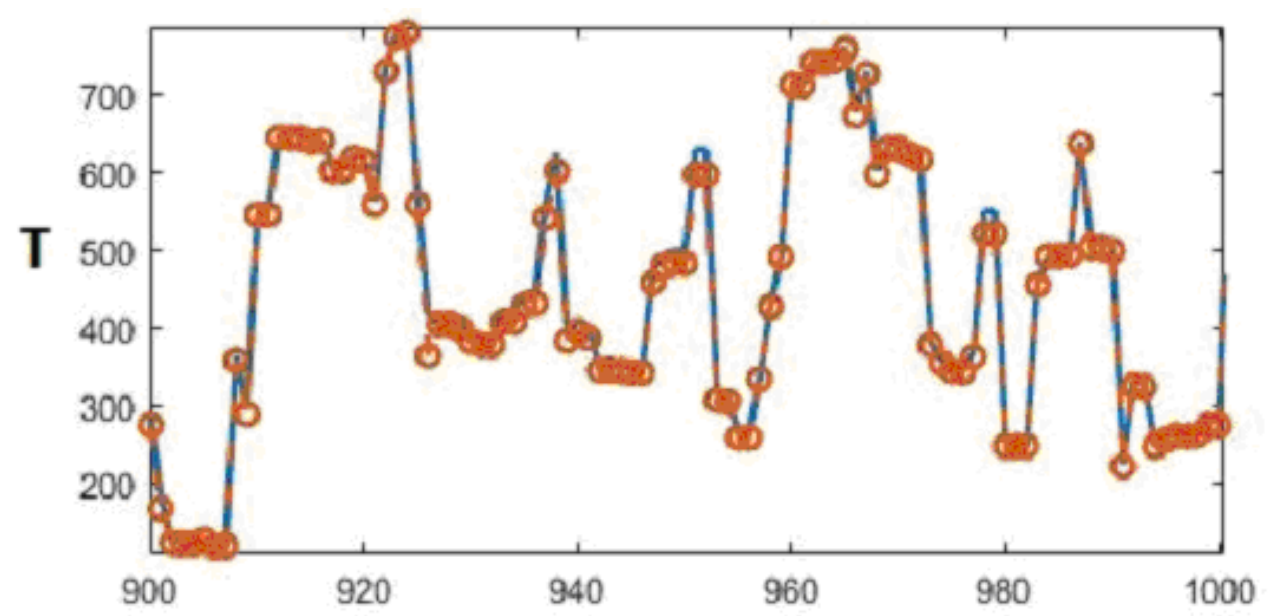

Figure 3. A randomly selected part of the graph of the temperature as a function of the space variable. The blue line is the high-precision solution while the orange circles are the values produced by the new algorithm for $h=0.0002$.

With the new "constant-neighbour" method, one timestep takes roughly $0.0004 \mathrm{~s}$. We found that if $h=0.0002$, then the produced results already fit quite well to the exact curve. The result is presented in Figure 3 . One can see that it is possible to obtain a reasonable solution in $t_{\mathrm{FIN}} \times 0.0004 \mathrm{~s} / h \approx 20 \mathrm{~s}$ (in reality, $14 \mathrm{~s}$ ), much faster than the conventional explicit or implicit methods.

In Table 1 some results obtained by MATLAB routines ode15s, ode23s and the new method, are summarised. We note that no matter how huge the error tolerance is set to enhance speed, we were not able to obtain any results in 3 minutes by any MATLAB routines. From the data one can see that when a larger error tolerance is set, the new method is at least comparable with the standard solvers. 
Table 1. Performance of 3 different solvers, $\mathrm{CN}$ is for "ConstantNeighbour". The first error quantity, MaxD, is the maximum deviation (absolute value of the difference) from the reference solution. The second one, SumD, is the sum of these deviations for all of the cells.

\begin{tabular}{|c|c|c|c|}
\hline Method & Runtime (s) & MaxD & SumD \\
\hline \hline ode15s & 181 & 7.7 & 4890 \\
\hline ode15s & 248 & 0.49 & 266.4 \\
\hline ode15s & 400 & 0.001 & 0.506 \\
\hline ode23s & 2112 & 0.146 & 79.6 \\
\hline $\mathrm{CN}, 2 \times 10^{-4}$ & 14 & 340.9 & 38702 \\
\hline $\mathrm{CN}, 2 \times 10^{-5}$ & 142 & 36.65 & 3570 \\
\hline $\mathrm{CN}, 10^{-4}$ & 307 & 15.75 & 1715 \\
\hline $\mathrm{CN}, 10^{-6}$ & 556 & 7.06 & 823 \\
\hline
\end{tabular}

On the other hand, if a high-precision solution is needed, then the proposed method could not be recommended, but first order methods are not for this purpose, anyway. However, the following facts should be emphasized:

- This is still a small system with 5000 cells, and for larger numbers of cells implicit methods have more serious drawbacks. We note that the reason we have not used larger systems for testing purposes is that our computer cannot solve them by implicit methods because of the huge memory requirements.

- This is the simplest, "raw" version of the new method, without any optimization, adaptive stepsize control, parallelization or vectorization, which could immensely enhance computing speed.

In Figure 4 we present the two different kinds of error as a function of the stepsize $h$. One can see that for smaller stepsizes, the errors are decreasing slightly faster than the stepsize, which underpins that the convergence-rate of the method is (at least) one.

It can be seen that the error function is almost horizontal at the right side of Figure 4. The reason for this is that there are tiny cells with very small time constant $\tau$, which means that their temperatures change very rapidly. In this case, the constantneighbour approximation we used in Section 2 step 1 is very crude for large time-step sizes and the method underestimates the speed of the processes in the system. The right side of the diagram also reinforces the statement that the method is stable for arbitrarily large step sizes, as the error does increase quite slowly with increasing stepsize and tends to a constant, thus they are always bounded and the method is stable. 


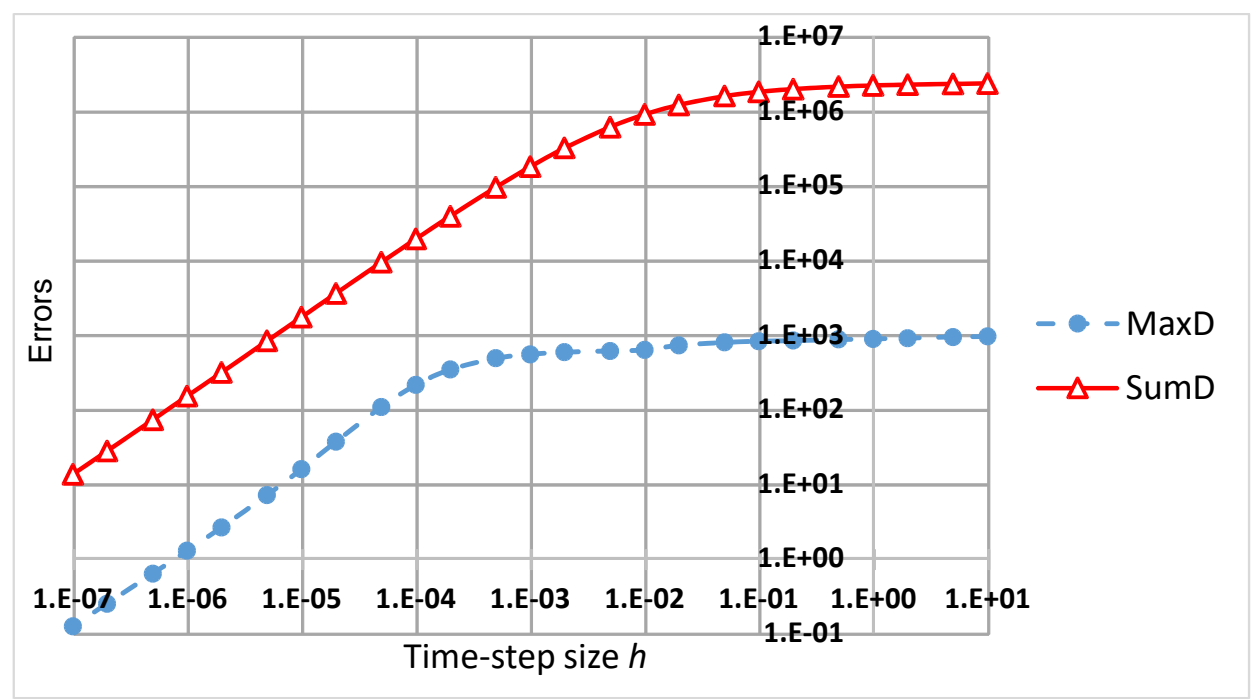

Figure 4. Different kinds of errors as function of the timestep size. For the definition of the error quantities, see the caption of Table 1 .

\section{SUMmary}

In this paper a new explicit numerical algorithm was presented to solve the timedependent heat conduction or diffusion equation with external sources. After the spatial discretization of these parabolic partial differential equations, one obtains systems of ordinary differential equations which consist of several equations and which can be extremely stiff. When applied to these equation systems, the new method is unconditionally stable and first order in time. The performance of the method has been illustrated for a simple, analytically soluble case and in the case of a twodimensional system with highly inhomogeneous random parameters. The obtained data suggest that if quick results are required for a huge number of cells, the new method has a remarkable advantage, even without parallelization. We have also proven analytically that the method is first order in time. We note that we are currently working on the second order version and also on extending the method to equations with other terms besides the diffusion term.

\section{REFERENCES}

1. U. M. Ascher, S. J. Ruuth, and B. T. R. Wetton. "Implicit-explicit methods for time-dependent partial differential equations." SIAM Journal on Numerical Analysis, 32(3), (1995), pp. 797-823. DOI: 10.1137/0732037.

2. Vabishchevich P. N. and Zakharov P. E. "Explicit-implicit splitting schemes for parabolic equations and systems." Lecture Notes in Computer Science, 2015. DOI: $10.1007 / 978-3-319-15585-2 \_18$. 
3. M. Cusini. "Dynamic Multilevel Methods for Simulation of Multiphase Flow in Heterogeneous Porous Media." PhD. Delft University of Technology, 2019. DoI: 10.4233/uuid:c624cd58-25e0-4bf9-bf36-025e08c46169.

4. E. Hairer and G. Wanner. Solving Ordinary Differential Equations II. Stiff and Differential-Algebraic Problems. Berlin: Springer-Verlag, 1991. DOI: 10.1007/ 978-3-319-15585-2_18.

5. M. S. A. Taj. "Higher Order Parallel Splitting Methods for Parabolic Partial Differential Equations." PhD. Brunel University, Uxbridge, England, 1995.

6. A. B. Gumel, W. T. Ang, and E. H. Twizell. "Efficient parallel algorithm for the two-dimensional diffusion equation subject to specification of mass." International Journal of Computer Mathematics, 64, (1997), pp. 153-163. DOI: 10.1080/00207169708804580.

7. G. Xue and H. Feng. "A new parallel algorithm for solving parabolic equations." Advances in Difference Equations, 20(1), (2018). DOI: 10.1186/s13662-0181617-8.

8. G. Martin, B. Bailey, and A. Piziali. ESL Design and Verification: A Prescription for Electronic System Level Methodology. Elsevier, 2010.

9. L. Skvortsov. "Explicit stabilized Runge-Kutta methods." Computational Mathematics and Mathematical Physics, 51(7), (2011), pp. 1153-1166. DOI: 10.1134/ S0965542511070165.

10. J. G. Verwer, W. H. Hundsdorfer, and B. P. Sommeijer. "Convergence properties of the Runge-Kutta-Chebyshev method." Numerische Mathematik, 57(1), (1990), 157-178. DOI: 10.1007/BF01386405.

11. M. N. Özisik, H. R. B. Orlande, M. J. Colaço, and R. M. Cotta. ESL Design and Verification: A Prescription for Electronic System Level Methodology. Boca Raton: CRC Press, 2010. DOI: 10.1201/9781315121457.

12. M. Hochbruck and A. Ostermann. "Exponential integrators." Acta Numerica, 19, (2010), pp. 209-286. DOI: 10.1017/S0962492910000048.

13. A. R. Gourlay and S. McKee. "The construction of hopscotch methods for parabolic and elliptic equations in two space dimensions with a mixed derivative." Journal of Computational and Applied Mathematics, 3,(3) (1977), pp. 201-206. DOI: $10.1016 /$ S0377-0427(77)80009-5.

14. E. Kovács and A. Gilicz. "New stable method to solve heat conduction problems in extremely large systems." Design of Machines and Structures, 8,(2) (2018), pp. 30-38. DOI: 10.1016/S0377-0427(77)80009-5.

Appendix A. The proof that the method is First order in time

Using the power series form of the exponential function, the exact solution of (1) is the following:

$$
\begin{aligned}
\vec{T}(t)=e^{M t} & \vec{T}_{0}+\left(e^{M t}-1\right) M^{-1} \vec{Q}= \\
& =\left(1+M t+M^{2} \frac{t^{2}}{2}+M^{3} \frac{t^{3}}{3 !}+\ldots\right) \vec{T}_{0}+\left(t+M \frac{t^{2}}{2}+M^{2} \frac{t^{3}}{3 !}+\ldots\right) \vec{Q} .
\end{aligned}
$$


The $0^{t h}$ and first order terms in the exact solution at $t=h$ are given by:

$$
\begin{aligned}
T_{\mathrm{i}}(h)=T_{0, i}\left(1+m_{i i} h\right)+h \sum_{j \neq i} m_{i j} T_{0, j} & +Q_{i} h= \\
& =T_{0, i}\left(1-\frac{h}{\tau_{i}}\right)+h \sum_{j=n e i g h} \frac{U_{i j}}{C_{i}} T_{0, j}+Q_{i} h,
\end{aligned}
$$

where we used the fact that $U_{i j} \equiv 0$ if and only if the two cells are neighbours and thus the summation can be performed only to the neighbours. Let us compare it to the new "Constant Neighbour" solution. We obtain from (3) that

$T_{i}(h)=T_{0, i}\left(1-\frac{h}{\tau_{i}}+O\left(h^{2}\right)\right)+\left(\frac{\sum_{j=n e i g h} U_{i j} T_{0, j}}{\sum_{j=n e i g h} U_{i j}}+Q_{i} \tau_{i}\right)\left(1-1+\frac{h}{\tau_{i}}-O\left(h^{2}\right)\right)$.

Considering only the $0^{\text {th }}$ and first order terms yields

$$
\begin{aligned}
T_{i}(h) \approx T_{0, i}\left(1-\frac{h}{\tau_{i}}\right) & +\left(\frac{\sum_{j=n e i g h} U_{i j} T_{0, j}}{\sum_{j=n e i g h} U_{i j}}+Q_{i} \tau_{i}\right) \frac{h}{\tau_{i}}= \\
= & T_{0, i}\left(1-\frac{h}{\tau_{i}}\right)+h \frac{\sum_{j=n e i g h} U_{i j} T_{0, j}}{\sum_{j=n e i g h} U_{i j}} \frac{\sum_{j=n e i g h} U_{i j}}{C_{i}}+Q_{i} h .
\end{aligned}
$$

After simplification we obtain the statement that the difference between the exact solution and the new method, i.e., the local error is second order in time. 